\title{
Repensar el cuidado a través de la migración internacional: mercado laboral, Estado y familias transnacionales en Ecuador ${ }^{*}$
}

\author{
Gioconda HERRERA MOSQUERA \\ Profesora Investigadora del Programa de Sociología \\ Facultad Latinoamericana de Ciencias Sociales (FLACSO-Ecuador) \\ gherrera@flacso.org.ec
}

Recibido: 17-09-2011

Aceptado: 24-11-2011

\begin{abstract}
RESUMEN
Este artículo examina la organización social de los cuidados entre familias transnacionales. Tomando el caso de la migración ecuatoriana a España indaga hasta qué punto la migración internacional ha significado cambios en esta organización en los contextos de origen de la migración. Para ello se examinan tres ámbitos: el mercado laboral de los cuidados, los servicios estatales en torno al cuidado y a las familias migrantes. El texto analiza los distintos ejes de desigualdad que atraviesan estos tres ámbitos y muestra cómo el género, la clase y la generación estructuran los arreglos y desarreglos del cuidado que se producen en la articulación de estas tres instituciones Sociales. El análisis se basa en los hallazgos de una investigación realizada en 2009 y 2010 en una zona periurbana de la ciudad de Quito.
\end{abstract}

Palabras clave: organización social de los cuidados, género, migración, familias transnacionales.

\section{Rethinking care through international migration: labour market, State and transnational families in Ecuador}

\begin{abstract}
This article examines the social organization of care among transnational families in Ecuador. It explores to what extent international migration has meant transformations in such care organization and examines three areas: the labour market of care in Ecuador, social policies around care and migrant family arrangements. The text looks at how gender, generation and class are inequality axes that cross these three areas and structure both institutional and family arrangements of care. The analysis is based on the findings of a research conducted in 2009 and 2010 in a peri urban area of the city of Quito.
\end{abstract}

Key words: social organization of care, gender, migration, transnational families

* Agradezco el apoyo de Iván Roa y de Consuelo Sánchez, becarios del programa de sociología de FLACSO Ecuador, quienes trabajaron en la sistematización de alguna de la información para este artículo. Una versión preliminar del mismo fue presentada en el XXIX Congreso de la Latin American Studies Association (LASA) en octubre de 2010 en Toronto. 


\section{REFERENCIA NORMALIZADA}

Herrera Mosquera, G., (2012). Repensar el cuidado a través de la migración internacional: mercado laboral, Estado y familias transnacionales en Ecuador. Cuadernos de Relaciones Laborales Vol. 30, núm. 1, p.139-159.

SUMARIO: Introducción. 1. Puntos de partida: organización social de los cuidados, reproducción social y manejo de los riesgos en la globalización. 2. La migración interna, intra regional y transcontinental de las mujeres en América Latina. 3. La organización social del cuidado: mercado, estado y familias. 4. El espacio familiar como espacio de cuidado: las dinámicas de las familias transnacionales. 5. Conclusiones. Bibliografía.

\section{Introducción}

El argumento del texto es que las dinámicas de cuidado producto de la migración, especialmente la femenina, reflejan procesos más generales de desigualdad social presentes en las realidades institucionales que sostienen las actividades de cuidado en la sociedad de origen. Así, más que un "déficit o drenaje de cuidados", lo que se producen son dinámicas complejas de arreglos y desarreglos que deben ser entendidas a partir de la especificidad histórica e institucional del trabajo reproductivo en cada país, en este caso en Ecuador. Pero además, la experiencia migratoria ha visibilizado determinadas prácticas de cuidado (o su ausencia) y al hacerlo ha servido para poner en discusión tres temas relevantes para una agenda de equidad de género: la diversidad de formas familiares, la importancia de las actividades reproductivas en la vida y desarrollo de las personas y, derivado de ello, la necesidad de pensarlas como un tema de agenda pública en sociedades donde la familia y las mujeres han sido históricamente el sostén de la reproducción. En ese sentido, la migración y sus efectos sobre las familias puede constituirse en una oportunidad para colocar en el centro de la discusión un viejo tema de los derechos económicos y sociales de las mujeres que es la desigual distribución entre trabajo remunerado y no remunerado, tema que había permanecido relativamente dormido en las décadas anteriores en la agenda feminista en América Latina.

El análisis se basa en los hallazgos de una investigación realizada en 2009 en una zona periurbana de la ciudad de Quito, que trató de reconstruir las prácticas de cuidado de las familias migrantes y su relación con la configuración institucional de la protección social en el Ecuador. Se realizó en primer lugar un mapeo de actores e instituciones encargados de actividades de cuidado en el país y luego un trabajo etnográfico en un barrio con alta incidencia migratoria en la ciudad de Quito para analizar la forma en que las familias se relacionaban con el mercado local de los cuidados, con el estado y de qué manera funcionaban las redes locales y transnacionales de cuidado. También se hizo un seguimiento pormenorizado a 9 familias transnacionales en Ecuador y en España. La caracterización del mercado laboral de los cuidados se basa en información cuantitativa proveniente de la Encuesta Nacional de Empleo, Desempleo y Subempleo del Ecuador-ENEMDU 2007 (INEC, 2007) y la Encuesta de Condiciones de Vida - ECV 2006 (INEC, 2006). La medición del 
trabajo no remunerado migrante y no migrante se basa en la Encuesta Nacional de Uso del Tiempo de Ecuador - EUT 2007 (INEC-Consejo Nacional de Mujeres, 2007) $)^{1}$.

El texto tiene cuatro partes: en primer lugar se presentan los puntos de partida conceptuales que orientan la interpretación, principalmente en torno a qué se entiende por organización social del cuidado y cómo se establece la relación entre globalización, migración y organización social del cuidado, y porqué es necesario mirar más allá de la idea de déficit de cuidados cuando se analizan los eslabones más débiles de las cadenas globales de cuidado: las sociedades de origen. Luego se exponen algunos rasgos del contexto histórico y del escenario actual de la migración femenina desde América Latina a Europa para mostrar que la migración, primero interna y luego internacional de las mujeres, ha sido parte de la experiencia histórica de reproducción social y de manejo de los riesgos en la vida de las familias andinas y de Ecuador. Así mismo, la maternidad a distancia y diversas nociones del cuidado son parte de estas construcciones sociales e históricas. Por ello, la reciente migración a Europa, si bien trastoca muchas prácticas sociales de cuidado tampoco es un fenómeno ajeno para las mujeres y sus familias. En la tercera sección, se examinan las condiciones del mercado laboral doméstico para las mujeres y en especial del mercado de los cuidados y el papel del estado y las políticas sociales en Ecuador respecto a las familias migrantes. Esto con el fin de mostrar que existe una dimensión constitutiva de género en la estructuración tanto del mercado de los cuidados como de las políticas estatales que marca la forma en que se establecen los vínculos entre trabajo remunerado y no remunerado. Esto afecta particularmente la condición social de las mujeres y puede marcar la opción de una reproducción transnacional. En la cuarta parte miro lo que ocurre en términos de la organización de los cuidados en las familias migrantes para mostrar que la migración exacerba realidades de desigualdad anteriormente existentes.

En último término, lo que intenta mostrar el texto es que es necesario mirar tanto el proceso histórico como la articulación de estas tres dimensiones institucionales estado, mercado y familias- para aprehender la complejidad de las dinámicas de cuidado en las sociedades de origen de las familias migrantes. En ese sentido, el concepto de cadenas globales de cuidado si bien tuvo la virtud de visibilizar lo que se denominó el último eslabón de la cadena, se queda corto al momento de captar

${ }^{1}$ El artículo se basa en un estudio que a su vez fue parte de un proyecto más amplio coordinado por el otrora Instituto para la Formación y la Investigación de la Mujer -INSTRAW-y que incluía el análisis de los flujos desde Amaría Latina a España y Chile. El proyecto se llamó "Mujeres latinoamericanas en las cadenas globales del cuidado". Agradezco a Amaia Pérez Orozco y Mar García, así como a las colegas de Bolivia, Perú y Chile por los comentarios en diferentes etapas de la investigación y que inspiraron indirectamente este artículo. 
las complejas dinámicas de cuidado presentes en las sociedades de origen de las familias migrantes.

\section{Puntos de partida: organización social de los cuidados, reproducción social y manejo de los riesgos en la globalización}

La migración internacional de mujeres del Sur hacia tareas de reproducción antes no necesariamente mercantilizadas en el Norte, ha reactualizado las discusiones del feminismo marxista de los años 1970 que, al enfatizar en la distinción entre trabajo productivo y reproductivo, buscaba subrayar la invisibilidad del trabajo femenino en el ámbito reproductivo y su importancia como sustento del bienestar y el sistema económico en general. En ese entonces, se trataba de entender las conexiones entre las condiciones de la participación de las mujeres en el mercado laboral y su concentración y responsabilidades frente al trabajo reproductivo; también se quería remarcar en el peso del trabajo no remunerado sobre la economía en general (Benería, 2008).

Todos estos elementos siguen siendo importantes en la discusión actual pero lo que estaría mostrando la migración internacional de mujeres hacia tareas reproductivas es que las fronteras entre trabajo productivo y reproductivo parecen haber cambiado. El trabajo reproductivo se ha mercantilizado aunque se realice en casas. Este nuevo contexto ha intensificado los problemas de las mujeres en cuanto a equilibrar el trabajo reproductivo con el del mercado y ha significado que algunas mujeres inclusive encuentren una resolución transnacional a esta articulación entre trabajo productivo y reproductivo (Benería, 2008).

Retomo la noción de reproducción social avanzada por Bakker y Gil (2003), entendiéndola como la transformación de los procesos sociales y los mecanismos, instituciones y prácticas necesarias para el sustento de las comunidades. Esto implica, fundamentalmente aunque no exclusivamente, al cuidado, además del proceso más general de sustento de vida. Dentro de este marco general, se trata de mirar cómo se organiza el cuidado socialmente con el fin de captar las jerarquías y desigualdades presentes en este proceso, tanto a nivel de las familias, como en relación al rol del estado y del mercado. En los países europeos, y en España en particular, los debates en torno a la organización social del cuidado han girado en torno a la interrelación entre el trabajo no remunerado ejercido por las familias, y las mujeres en particular, su reciente mercantilización en manos de mujeres migrantes, las transferencias monetarias a las familias por parte del Estado y/ o los servicios sociales que ofrece el estado para determinadas tareas. El distinto balance que se produce entre estos factores refleja las jerarquías del sistema y el peso cada vez mayor de la fuerza de trabajo inmigrante en estas actividades (Vega, 2009; Martínez Buján, 2011).

En América Latina, con un estado mucho menos presente, estos procesos toman otra figura. Para analizarlo me sirvo de la noción adelantada por Martínez Franzoni (2008) sobre el bienestar en tanto capacidad de manejo de los riesgos sociales - 
entiéndase- el cuidado de niños y personas mayores, las enfermedades, las crisis, la discapacidad, antes que como un estado de situación. Para esta autora, en América Latina esta concepción del bienestar ha significado en muchas ocasiones incertidumbre frente a los riesgos. En ausencia del estado, estos han sido manejados mediante mecanismos que permiten menos su prevención y más su amortiguamiento. En efecto, idealmente podríamos contar con tres tipos de mecanismos, según la misma autora: los formales mercantiles que se cumplen a través de los ingresos y ciertas políticas públicas, tales como subsidios, la generación de empleo, la definición de estándares laborales o informales, y la autoprotección que son las prácticas que despliegan las personas de manera informal a través de las redes y sirviéndose de determinados activos.

Pero los riesgos no son individuales sino que se construyen socialmente. La clase y el género son dimensiones de estratificación social que definen la estructura de los riesgos con las que las personas y sus familias se enfrentan. Por ello es necesario mirar distintos escenarios donde se construyen estos riesgos (Martínez Franzoni, 2008). Ante Estados que generalmente han fallado en asignar recursos suficientes para el manejo de los riesgos son las familias las que recurren a diversas prácticas mercantiles y no mercantiles en contextos altamente cambiantes.

En efecto, la presencia de las mujeres en el mercado laboral ha aumentado drásticamente en los últimos 10 años en América Latina y se explica principalmente como una estrategia frente a la pobreza y a la desigualdad antes que como producto de cambios en los modelos deseables del mercado y la organización familiar. Por otra parte, la organización social de la reproducción social acontece en escenarios altamente estratificados económica, socialmente y en términos de género: hay personas y familias con múltiples opciones para el manejo de los riesgos y otras con muy pocas y por tanto coexisten diversos mundos de reproducción social en un mismo territorio (Martínez Franzoni, 2008). La migración internacional se convierte en estos escenarios en una estrategia de manejo de los riesgos, pero a su vez estaría produciendo nuevos riesgos.

Más allá de la importancia nada deleznable de las remesas para los hogares, que ha sido la manera predominante de entender la relación entre migración y reproducción social, lo que se busca comprender aquí son las dinámicas de la organización social de los cuidados en las familias migrantes, en tanto manejo de los riesgos, es decir de manera más integral.

Pero además, las prácticas de cuidado están arraigadas en ideologías de género y concepciones hegemónicas sobre la familia y el cuidado que circulan a través del discurso público y en espacios de interacción social que otorgan significados a lo que funciona normativa y culturalmente como aceptable y pertinente. Por ello, el análisis de las estrategias frente al cuidado de las familias migrantes tiene que ir más allá de las estrategias de reproducción económica y social e investigar los significados - las tensiones y contradicciones - que se derivan de estas prácticas para los miembros de los hogares y las comunidades (Pribilsky, 2007).

Sin embargo, la migración internacional como opción de reproducción social transnacional no puede ser atribuida a factores domésticos únicamente - léase la 
crisis económica o la falta de políticas sociales de manejo de los riegos en los países de origen- sino que está ligada a procesos globales de carácter estructural que tienen que ver con formas de acumulación de capital basadas no sólo en la transnacionalización del capital o de la producción sino también de la reproducción (Bakker \& Gill, 2003). En ese sentido se trata de "opciones" estructuradas en un sistema global en el que, tal como lo ha expresado Hondagneu-Sotelo (2001) las actividades de sustento de la vida relativas al cuidado están siendo mercantilizadas cada vez más en manos de mujeres inmigrantes del Sur. Esto ha dado lugar a lo que diferentes autoras han llamado la privatización o la informalización de los servicios del cuidado a nivel global (Bakker \& Silvey, 2008; Hondagneu-Sotelo 2001; Salazar Parreñas, 2001) o al surgimiento de uno de los conceptos más populares en el análisis de la migración internacional de mujeres que es el de las cadenas globales de cuidados (Ehrenreich \& Hochschild, 2003).

Acuñado en 2001 por Ehrenreich y Hotchschild (2003) el concepto de cadenas globales de cuidado ha sido ampliamente utilizado para explicar la migración de las mujeres en distintas regiones del mundo y con ellas el desplazamiento de las actividades reproductivas, a.i. de los cuidados del Sur al Norte. Es decir que conjuntamente con los flujos de capitales, de información y de mercancías que circulan en la globalización también se producen circuitos internacionales de cuidadoras que garantizan la reproducción social de muchos seres dependientes e independientes en todo el planeta. Estos circuitos fueron analizados en la migración de mujeres asiáticas al medio Oriente, a Europa y Estados Unidos primero y más tarde, con el boom se convirtieron en una de las explicaciones más interesantes a la hora de analizar el súbito crecimiento de la migración femenina andina a Europa ${ }^{2}$. La idea básica detrás del concepto es mostrar que mientras más se desciende en la cadena, el valor del trabajo decrece y poco a poco se convierte en no remunerado (Yeats, 2005). Además las transferencias de cuidado atraviesan ejes de desigualdad complejos que incluyen relaciones de género, clase y raza (Pérez Orozco, 2009) pero también inter generacionales, geopolíticos y otros.

El concepto constituyó una herramienta muy útil para conectar las realidades estructurales de las sociedades de origen y de destino en la experiencia migratoria y analizar el carácter asimétrico de cada una de las transferencias que la cadena sostiene, mostrando que estas transferencias implican desnaturalizar al trabajo doméstico no remunerado y colocarlo como un eje articulador que explica la razón de ser de estas cadenas a nivel nacional e internacional y por tanto colocar las desigualdades de género como articuladoras de las otras formas de desigualdad.

${ }^{2}$ En la revisión que Yeats (2005) establece al concepto se describe a la cadena global de cuidado prototípica con el siguiente ejemplo: "Una hermana mayor de una familia de bajos recursos que cuida de sus hermanas mientras su madre trabaja como niñera cuidando a otro niños cuya madre ha migrado y quien, a su vez cuida a los niños de una familia en un país rico" (Yeats, 2005). 
Si bien fueron muchas las bondades del concepto para entender las matrices de género presentes en esta globalización de la reproducción social en manos de mujeres migrantes, algunos trabajos han empezado a interrogar este concepto desde distintas aristas. Por un lado, se señala que las cadenas han analizado fundamentalmente la relación de un solo tipo de migración femenina y un solo tipo de cuidados, el de madres migrantes que cuidan a menores mientras el cuidado de sus propios hijos es ejercido por una tercera persona en otro país. Sin embargo, la realidad tanto de los cuidados como de las cuidadoras es mucho más diversa e involucra no sólo a mujeres madres sino a profesionales de la salud, cuidadoras de adultos mayores y muchos más (Williams 2010; Yeats, 2005). Además, Williams (2010) señala que es necesario comprender esta relación cuidadores/cuidados en el marco más amplio de una economía política global del cuidado, que incluya en el análisis el movimiento de los trabajos de cuidado, pero también el de los compromisos de cuidado (maternidad transnacional), la mercantilización transnacional del cuidado y la transnacionalización de los discursos y políticas de cuidado. Este marco permite entender que las cadenas de cuidado se desenvuelven en realidades institucionales e ideológicas mucho más amplias que las estructuran. Por último, la idea de "déficit o drenajes de cuidado" parece encontrar sus límites a la hora de examinar detenidamente los elementos que entran en juego en el análisis de la organización social del cuidado entre familias migrantes en los países de origen. En efecto, una mirada detallada a las percepciones y prácticas en torno a los arreglos y negociación de los cuidados implica rescatar, por un lado, la agencia de las mujeres migrantes que permanecen en contacto con sus familiares en origen, y por otro lado, las acciones desplegadas por las redes familiares y sociales. Pero además, en la configuración de esta organización social del cuidado en la migración se redefinen nociones sobre el cuidado, la maternidad, la identidad de las mujeres migrantes, de las y los cuidadores, y de las personas que demandan cuidados. Es decir, más allá de las transferencias desiguales de cuidado, las prácticas que se activan y desactivan con la migración reflejan procesos de desigualdad social al interior de estas redes. Por ello, interesa también subrayar que las actividades de cuidado no siempre se reparten de manera igualitaria entre las personas, entre las familias, entre hombres y mujeres, entre mujeres de diferentes clases sociales, y no sólo entre países. De ahí que sea necesario complementar esta geografía de la desigualdad global con la reconstrucción de la historia de estas redes locales de cuidado y la forma cómo éstas están atravesadas por ideologías de género. A continuación, presento brevemente la experiencia migratoria en América Latina para luego analizar la organización social del cuidado entre las familias migrantes. 


\section{La migración interna, intra regional y transcontinental de las mujeres en América Latina}

La migración de las mujeres en América Latina no es un fenómeno nuevo. Durante la época colonial, muchas mujeres de zonas rurales fueron a las ciudades, principalmente como trabajadoras domésticas, ya sea como migrantes permanentes o temporales (Kusnesof, 1989). Las mujeres eran también una parte importante de los flujos rural-urbano que prevalecieron durante los siglos XIX y $\mathrm{XX}$ en el continente. En algunos períodos, las mujeres jóvenes, eran incluso las principales protagonistas de estos flujos (Ariza, 2000; Pellegrino, 2003). De hecho, la migración era a menudo el mecanismo por el cual muchas mujeres pobres de América Latina entraron por primera vez al mercado laboral, por lo general en el servicio doméstico (Ariza, 2000: 17). La gran mayoría de estas mujeres eran jóvenes y solteras y con frecuencia transgredían los roles de género y las ideologías con su experiencia migratoria. La maternidad a distancia también es parte de la historia de la migración de las mujeres en América Latina. La crianza y reproducción de niños y adultos mayores a través de su trabajo en las ciudades o en el extranjero no era un fenómeno desconocido en las zonas rurales de muchos lugares de América Latina (Kusnesof, 1989).

Estudios sobre migración interna de las mujeres en el Ecuador y en la región andina atestiguan el hecho de que, en ausencia de las madres y padres, diversos arreglos familiares fueron desplegados a nivel local, no sin despertar algunas tensiones y una sobrecarga de trabajo para las mujeres que se quedaban (Poeschel, 1985; Rosero, 1986).

En la segunda mitad del siglo XX, la movilidad transfronteriza de las mujeres se convirtió en una extensión de la migración interna. Argentina y Venezuela fueron destinos importantes para los hombres y mujeres de los países andinos. La migración de las mujeres colombianas a Venezuela y mujeres del Paraguay a la Argentina se inició en la década de 1950 y continúa hasta hoy. A partir de 2000, en paralelo con el aumento importante de la migración transcontinental a Europa, la migración dentro de la región también creció considerablemente (Cerrutti, 2009). Los análisis de las condiciones de estas mujeres migrantes indican que su situación combina una precaria inserción laboral con discriminación étnica y racial. Por lo tanto, clase y raza refuerzan las desigualdades de género profundizando las condiciones de exclusión social (Magliano, 2007; Cerrutti, 2009; Stefoni, 2002). En la mayoría de los casos, la proximidad a su país de origen permite a estas mujeres mantener fuertes lazos de reproducción con sus familias y comunidades cuando no emigran con sus familias.

En los últimos quince años la geografía y composición de la migración internacional desde América Latina se ha transformado. Europa y España e Italia en particular, se han convertido en los principales destinos de los y las latinoamericanas y especialmente de las mujeres de la región andina. Además, el patrón tradicional de la migración masculina rural ha ido cambiando por un importante flujo de mujeres de origen urbano, muchas de ellas con perfiles educativos medios pero con magras 
perspectivas de movilidad social ascendente. Este rápido aumento de la migración andina a Europa y su patrón femenino han sido analizados en los últimos años desde diversas aristas. Los estudios han destacado una amplia gama de factores que van desde las explicaciones estrictamente económicas de búsqueda de oportunidades en otros mercados laborales hasta aquellas que matizan las decisiones migratorias de las mujeres con aspectos relativos a otros tipo de discriminaciones - étnicas, raciales, de género -, y también con la búsqueda de expectativas de cambio al imaginar mundos diferentes. (Camacho, 2009; Lagomarsino, 2007; Herrera, 2008; Hinojosa, 2009; Carrillo \& Cortés, 2008; Pedone, 2006; Bastia, 2009).

Uno de los destinos más importantes de los flujos ecuatorianos y andinos ha sido España. De acuerdo a los datos del Padrón Municipal, las personas ecuatorianas en España ascienden a 451.072 al año 2008 , de las cuales 219.080 son varones y 231.992 mujeres, o sea el $51,43 \%$.

Respecto a los procesos de reunificación familiar, diversos estudios han mostrado que si bien las mujeres fueron muchas veces las pioneras de las trayectorias migratorias de las familias, en el caso ecuatoriano estos procesos se produjeron con relativa rapidez (Camacho, 2009; Pedone, 2006; Herrera, 2008). Los datos demográficos confirman esta situación pues en las pirámides de población entre los años 2001 y 2008 es claro el predominio de las edades centrales, ya que el $83.78 \%$ de los ecuatorianos tiene entre 15 y 64 años. Pero en 2008 la proporción de niños y adolescentes ecuatorianos se incrementa hasta representar el $15.61 \%$ de la población expresando un acelerado proceso de reunificación familiar del flujo.

Respecto a la inserción laboral de los y las ecuatorianas en España, destaca el trabajo doméstico entre las principales ocupaciones de las mujeres. De acuerdo a la encuesta de Empleo, subempleo y desempleo del Ecuador - ENEMDU 2007, mientras en Ecuador esta ocupación le pertenecía al 4.8\%, en España le corresponde al $38 \%$. Las categorías restantes no muestran grandes variaciones salvo la de patronos/cuenta propia que pasa de $35.6 \%$ en Ecuador, a $4.1 \%$ en España. Los trabajos de Camacho (2009), Wagner (2009) y Herrera (2008) han mostrado que existe cierta movilidad al interior del trabajo doméstico, desde la condición de internas hasta el trabajo por horas en empresas de limpieza donde el carácter de empleada de hogar ya desaparece. Inclusive, a más años de migración mayores posibilidad de salir del nicho del trabajo doméstico. La crisis actual, sin embargo, ha modificado este patrón de cambio de sector laboral.

Respecto a los dependientes que han quedado en Ecuador, de acuerdo a la encuesta ENEMDU 2007, el $34.7 \%$ de las mujeres y el 38.8\% de los hombres han dejado al menos un hijo menor de dieciocho años en Ecuador. Es decir, no podemos hablar de un patrón definitivo de asentamiento de las familias en destino pues todavía quedan muchos hogares con al menos un hijo/a dependiente en origen. Eso significa que los vínculos en torno al cuidado entre estas familias son todavía muy relevantes, diez años después de la salida.

Los hogares migrantes en Ecuador se asientan en comunidades rurales y urbanas, que difieren en muchas cosas, entre otras en el nivel de acceso a los servicios públicos. Es importante señalar que el perfil de las personas que han salido en la 
última ola y de las familias que se han quedado se levanta sobre importantes clivajes de desigualdad, rural-urbanos, étnicos, y por supuesto de clase. Como veremos a continuación, la organización social del cuidado y su relación con la migración internacional responden a estos clivajes también.

\section{La organización social del cuidado: mercado, estado y familias}

Durante los años 1980 y 1990 las economías y las sociedades latinoamericanas se sometieron a varias pruebas de la política neoliberal que acentuaron la concentración de la riqueza y la desigualdad social. Más allá de las diferencias entre país y siguiendo el modelo de regímenes de bienestar en América Latina propuesto por Martínez Franzoni (2008), Ecuador es un régimen de bienestar informal-familista, caracterizado por niveles altos de informalidad del mercado laboral, especialmente cuando miramos la participación de las mujeres, por el acceso de grupos importantes de su población a mercados laborales trasnacionales como estrategia de reproducción y por la debilidad del estado en la creación de capacidades para manejar los riesgos sociales. Por tanto, la organización social del cuidado recae principalmente en las familias y en su capacidad de mercantilizar o de activar otros mecanismos no mercantiles para asegurar la reproducción (Martinez Franzoni, 2008).

Por otra parte, el panorama del mercado laboral para las mujeres en Ecuador en los últimos 15 años da cuenta de una profundización de la precariedad, de la segmentación laboral y un crecimiento en las brechas salariales entre hombres y mujeres. Para algunas autoras, esta ha sido un factor fundamental a la hora de tomar la decisión de emigrar (Camacho, 2009). La participación femenina en el mercado laboral en Ecuador creció de manera pronunciada en los último veinte años, pasando de $38 \%$ en 1988 a $48 \%$ en 1998 y 52,37\% en el 2006 (de acuerdo a ECV 2006), no así la calidad del empleo o los sistemas protección social que cubren los aspectos reproductivos. De la población ocupada, un gran porcentaje está subempleada, (64\% de la Población Económicamente Activa). Este afecta en mayor medida a las mujeres y es más alto entre la población indígena (Armas, 2008, en base a ECV 2006).

Por otro lado, el desempleo de las mujeres es más del doble del identificado para los varones y afecta más a las jóvenes de 18 a 29 años, a la población afro ecuatoriana y a las áreas urbanas (Armas, 2008). Además, el porcentaje de mujeres que trabaja en jornadas completas es muy bajo, no existen políticas o programas de conciliación entre el trabajo no remunerado y el remunerado, ni políticas que promuevan la corresponsabilidad social y de género en el trabajo reproductivo. (Genta $\&$ Contreras, 2010). Además, al estar más representadas en el sector informal, se enfrentan a deficiencias en los servicios de cuidado diario y de otros servicios públicos relativos al cuidado de las personas.

Los trabajos del cuidado no solamente no son valorados social y económicamente cuando se los realiza en el ámbito privado sino que también reciben una menor valoración, y por lo tanto menores remuneraciones, en el mercado laboral. Los 
trabajos tradicionalmente femeninos como el trabajo doméstico remunerado y en el sector de salud y educación, son ejemplos de esta subvaloración. Además, estos trabajos están vinculados a sectores de baja productividad. Por otra parte, mientras las mujeres están sobre-representadas en el empleo doméstico y las trabajadoras familiares no remuneradas $(95.81 \%$ y $63.69 \%$ respectivamente), las categorías de patrón $(77,16 \%)$ y asalariado privado $(63,29 \%)$ son masculinas con las consecuentes diferencias en términos de ingresos y condiciones laborales generales (Genta \& Contreras, 2010).

Así mismo, las brechas de ingresos entre varones y mujeres responden a los patrones de segregación horizontal y vertical. A estos patrones sexistas deben agregarse en Ecuador la marcada discriminación étnica que refuerza la segregación en tanto las poblaciones indígena y afro ecuatorianas son las que reciben menores remuneraciones. ${ }^{3}$. Cuando se toma en consideración los niveles de instrucción, las mujeres tienen un ingreso laboral menor al de los varones en todos los niveles. Pero además, esta brecha se incrementa en los niveles más altos de instrucción.

Respecto a las actividades de cuidado, estas son feminizadas, de bajos salarios, sin cobertura de seguridad social e inestabilidad. Así el 95.5\% del servicio doméstico, el $67,92 \%$ de las ocupaciones en actividades comunitarias y sociales, el 71,79 de los servicios sociales y de salud, y el 60,75 de las actividades de enseñanza son ocupadas por mujeres (ECV 2006).

En lo relacionado a la protección social, el servicio doméstico y las actividades comunitarias, sociales y personales son las que presentan las cifras más bajas de cobertura mientras que la enseñanza y la salud presentan una ventaja en este sentido, producto de la incidencia del empleo público.

Es decir, la situación de las mujeres en el mercado laboral ecuatoriano muestra que si bien ha crecido su incorporación al trabajo remunerado las diferencias señaladas indican significativas inequidades de género. Estamos frente a un panorama estructural de precariedad que afecta a las mujeres en general, a las mujeres en el sector de los cuidados en particular, y a las mujeres indígenas y afro descendientes de manera especial. Frente a estas limitaciones de los mercados domésticos no es de extrañarse que los mercados laborales transnacionales se conviertan en una opción de reproducción para las mujeres y las familias.

A estas precariedades se suma un rol del estado muy magro en crear las condiciones para cubrir las tareas reproductivas de las mujeres insertas en el mercado laboral. En efecto, si miramos la oferta estatal de servicios de cuidado la tendencia de los últimos años ha sido invertir cada vez más en políticas focalizadas. Actualmente, la política social en Ecuador tiene dos componentes principales: entrega de beneficios sociales universales permanentes a través de acciones secto-

${ }^{3}$ El ingreso medio de las mujeres indígenas es de 110 dólares mientras que el de los varones blancos es de 429,300\% más. A nivel nacional, el ingreso promedio de las mujeres representa el 78\% del ingreso de los varones (Genta \& Contreras, 2010). 
riales y los programas de asistencia social específicos focalizados en los más pobres ${ }^{4}$. En el periodo 1995-2008 no se han establecido cambios sustanciales en la orientación de los programas sociales que venían operando desde la década anterior. Al contrario, se ha enfatizado en la asignación presupuestaria a los programas focalizados: estos programas se dirigen a población pobre y se circunscriben a proyectos de alimentación y algunos de educación ${ }^{5}$.

Respecto al sujeto de la política, ciertas políticas están direccionadas a las mujeres en tanto madres: el Bono de Desarrollo Humano se dirige a mujeres madres, el programa de alimentación y nutrición (PANN) y el de Aliméntate Ecuador se basa en el trabajo de las "madres comunitarias", mientras que la Ley de Maternidad Gratuita tiene como población beneficiaria a las mujeres embarazadas y en edad fértil. Así, la privatización o mercantilización del cuidado obedece a que la oferta estatal para edades tempranas es insuficiente. Las escasas medidas están dirigidas a las mujeres en tanto cuidadoras en una serie de políticas sectoriales como las de trabajo y previsión social (Marco, 2006).

En lo que refiere a las políticas dirigidas a los adultos mayores, estas son incipientes dentro del espectro más amplio de las políticas públicas hacia la población infantil y juvenil, las mujeres y la población con discapacidades. Las personas mayores se encuentran aún en una situación más precaria, ya que constituyen todavía un grupo relativamente invisible en el mundo de las políticas públicas, con el agravante de su creciente participación demográfica, la disminución de sus ingresos y el aumento de la pobreza y la inseguridad en todos los ámbitos de la vida (Genta, $2011)^{6}$.

En definitiva, dos rasgos resumen la oferta de cuidados entregada por el estado, ecuatoriano: se trata de una oferta focalizada, con baja cobertura y cuya concepción

\footnotetext{
${ }^{4}$ Entre los universales, los de mayor importancia son la Ley de Maternidad Gratuita y Atención Infantil (producto de una ley de 1994 y reformada en 1998) y el Aseguramiento de Salud. Mientras que en las focalizadas se destacan el Bono de Desarrollo Humano - BDH (antes Bono Solidario) y el Programa de Alimentación y Nutrición (PANN 2000). El BDH es el programa de transferencias monetarias más grande de la región, representando el $0,6 \%$ del PIB en el 2006 y el $0,84 \%$ en el 2007 y el $72 \%$ de todos los recursos entregados. El otro gran programa de transferencias monetarias, Oportunidades (México), llega al 0,4\% del PIB (Grupo Faro, 2007).

${ }^{5}$ Con excepción de la Ley de Maternidad Gratuita, todos los programas están concentrados en la atención a los primeros dos quintiles o en los cantones más pobres del país. La mayor parte tiene como población objetivo a los niños y adolescentes (pobres) mientras que sólo uno está destinado a discapacitados y tres a adultos mayores.

${ }^{6}$ Sin embargo, en la Constitución Política de la República del Ecuador del año 1998 se elabora una legislación específica provista por la Ley Especial del Anciano de 1991, su Reglamento General de 1992 y las modificaciones posteriores. A pesar de esto, es notorio que la sociedad en general, desconoce los beneficios que promueve esta ley (Egüez: 2005:80).
} 
construye a las mujeres madres como intermediarias del bienestar de sus hijos. En estas políticas las mujeres se ciudadanizan a través de su rol de madres, esto les faculta a convertirse en receptoras e interlocutoras de la acción del estado (Genta \& Contreras, 2010).

Así, la mayor parte del cuidado se resuelve en la oferta privada, con serias consecuencias no sólo para las mujeres como sus principales responsables, sino también para los receptores del cuidado que se ven así sin el cuidado integral necesario (Marco, 2006).

Por otra parte, las iniciativas por parte del estado de apoyo a las familias con algún miembro migrante no tienen una amplia cobertura y consisten mayoritariamente en apoyos psicosociales, sin cubrir demandas de cuidados. Esto se debe al carácter focalizado de las políticas sociales que excluye a las familias migrantes en tanto potenciales receptoras debido a varios factores. Por un lado se privilegia a los quintiles uno y dos de ingresos, es decir a las familias más pobres; en segundo lugar, existe una cuasi exclusiva orientación de estas políticas hacia los niños y niñas, producto de la presencia desde hace casi 100 años de una visión maternalista de la política social. Y esto vuelve complejo pensar al cuidado y la protección social de los más dependientes como un tema universal.

Consecuentemente, las familias se han visto abocadas a la búsqueda de arreglos de cuidado desde el mercado o las redes sociales y familiares, antes y después de la migración internacional. Así mismo, la migración de las madres es interpretada por las instituciones del Estado dentro de este marco históricamente construido, colocando a los niños como sujetos principales de las políticas y las madres como los intermediarios de su bienestar. Por lo tanto, su ausencia se traduce automáticamente en la visión del estado en un déficit de cuidado.

En otras palabras, lo que esta organización segmentada del cuidado implica para las familias es que ante la ausencia del estado muchas familias buscaron la migración como forma de garantizar la reproducción social, y ahora que surgen nuevas necesidades de cuidado, tampoco está presente el estado.

\section{El espacio familiar como espacio de cuidado: las dinámicas de las familias transnacionales}

En contraste con los discursos sobre la familia fracturada y vulnerable que predomina tanto en las políticas como en el imaginario de las personas, las prácticas desplegadas por las familias migrantes para hacer frente al cuidado de niños y adultos mayores muestran un interés activo por parte de los miembros migrantes en el extranjero para mantener relaciones sostenidas con sus familiares de origen, y una serie de estrategias por parte de miembros de la familia en origen para hacer posible las actividades de cuidado.

Frente a la escasa presencia del estado en la organización social del cuidado queda por analizar cuál es rol que cumplen las familias en estas actividades y de qué manera lo hacen. Para ello es posible revisar la Encuesta del Uso del Tiempo (EUT 
2007) que fue aplicada a nivel nacional y además tiene la ventaja de contar con un módulo aplicado a familias con algún miembro migrante. De acuerdo al trabajo de Vásconez, Armas y Contreras (2009) las prácticas de cuidado entre familias con y sin migrantes no parecen experimentar grandes cambios. A pesar de la débil presencia del estado y de la baja mercantilización de los servicios entre las familias migrantes, la distribución de los tiempos de cuidado no parecen sufrir grandes variaciones entre los géneros al interior de las familias, confirmándose la persistencia de un orden de género que coloca en manos de las mujeres las principales responsabilidades frente al cuidado.

Sin embargo, si miramos los segmentos de edad de las cuidadoras encontramos una participación importante de mujeres menores de 18 años y mayores de 46 entre las familias migrantes, apuntando a la necesidad de poner más atención en qué está sucediendo con estos segmentos poblacionales en los arreglos del cuidado. Por otra parte, se vislumbra también un tímido incremento de la participación masculina en labores de cuidado en hogares migrantes que daría cuenta de algunas modificaciones en la tradicional distribución de los cuidados. Pero las mujeres -esposas, madres, hijas- son las principales dadoras de cuidados, tanto a nivel familiar como en la figura de madres comunitarias de los programas sociales. Es decir, las mujeres, a nivel privado y público son reconocidas como cuidadoras pero no como personas en necesidad de cuidados cuando algunas de ellas si lo son, tal como lo demuestra el trabajo cualitativo de la investigación. En conclusión, la distribución sexual del cuidado no parece variar con la llegada de la migración en cuanto a trabajo no remunerado se refiere.

Esto explicaría porque el envío de remesas por parte de las mujeres migrantes a sus madres o hijas cuidadoras, es en alguna forma un reconocimiento a su trabajo de cuidado, aunque no se lo explicite y se lo siga tratando como una "ayuda". Puesto que la accesibilidad a cuidados desde el mercado está directamente relacionada con el ingreso de las familias, las remesas pueden constituirse en una fuente importante de provisión de cuidados.

Veamos a continuación con más detalle, a través de los hallazgos del trabajo cualitativo de qué manera se resuelve la organización social de los cuidados en estas familias y qué jerarquías de género, de clase y generacionales, atraviesan esta organización.

En los casos de migración trasnacional reconstruidos en el estudio todas las mujeres migrantes iniciaron su inserción laboral en España en trabajos de cuidado. El trabajo en calidad de internas a cargo ya sea de niños o de ancianos les permitió enviar remesas para garantizar el sustento cotidiano de sus familias, el cuidado diario de adultos mayores o la educación de sus hijos e hijas. Es decir el cuidado de otros garantizaba el cuidado a distancia de los suyos. Poco a poco sin embargo, las actividades de trabajo doméstico en destino son remplazadas por trabajos en distinto tipo de servicios. Si bien el cuidado es uno de los oficios que se mantiene, las relaciones y situaciones laborales se modifican y en la mayoría de los casos ya se ha dejado atrás el Régimen Laboral Especial de trabajadoras del hogar a favor del Régimen General. Es decir, que la inserción laboral de las ecuatorianas en el lapso 
de diez años, ha tendido a modificarse rápidamente. De allí que las cadenas globales de cuidado si bien captan una realidad estructural fehaciente deben ser matizadas a la hora del análisis de las trayectorias migratorias y laborales de las personas como ya lo han señalado otras autoras (Yeats, 2005, Williams, 2010). Por eso, en el caso de la migración que nos ocupa, lo que los itinerarios laborales muestran es una serie de incursiones en el mercado de los cuidados muy variable. Más bien, la inestabilidad de los trabajos, su flexibilidad y su precariedad vuelven estos encadenamientos muy volátiles y ello afecta las formas de reproducción social en origen.

En cuanto a los cuidados en origen, en general, cuando se ha evaluado los impactos de la migración sobre las familias, se tiende a mirar únicamente la situación de quién es cuidado o falto de cuidados, esto recae generalmente en los y las niñas, dejando de lado otros actores. Uno de los hallazgos al momento de mirar estas redes familiares y locales a nivel micro es que los cuidados son relaciones sociales con varios "implicados", y es necesario tomar en cuenta no solo a la gente que necesita cuidados (niños, adultos mayores, personas dependientes) sino también ver simultáneamente la relación entre quién cuida y quién es cuidado. En determinadas situaciones se invisibiliza una de las partes de esta relación, en otras oportunidades esta diferencia entre cuidadores y cuidados no es tan clara. Una mirada relacional al cuidado permitió analizar los dos polos del vínculo, cuidadores y cuidados, y la interrelación que se establece entre ellos. Este es el caso por ejemplo de abuelas a cargo de adolescentes mujeres, relación en la cual muchas veces la adolescente hace de cuidadora en la cotidianidad aunque formalmente la cuidadora es la abuela. Encontramos que en varias ocasiones estos roles convergen en una sola persona, encontrando abuelas y nietas que son al mismo tiempo cuidadoras y cuidadas por ejemplo. Es decir la desigual distribución de los cuidados está cruzada por estas pautas sociales y culturales relativas al género y la generación.

En cuanto a los procesos de mercantilización de los cuidados encontramos que es un proceso bastante precario, que frecuentemente se engarza en redes sociales y de parentesco que tienden oscurecer el carácter de relación laboral presente en esta actividad. Así, encontramos prolongadas jornadas laborales, flexibilidad en el reconocimiento de honorarios de acuerdo a la ley, sobrecarga de trabajo. De alguna manera se reproduce y confirma el carácter subvalorado del trabajo de cuidado y una muy tenue división entre espacio público y privado, cuando éste es remunerado. Es importante señalar que tratándose de familias empobrecidas, la mercantilización del cuidado aparece como última opción, ya cuando el proyecto migratorio ha resuelto otros aspectos como la vivienda, los estudios y la salud. Además, lo que observamos es una mercantilización de las redes sociales y/o de parentesco más que un ingreso al mercado autónomo y anónimo. Es decir, se envían remesas para cubrir el cuidado que ejercen parientes y vecinas, por tanto siempre existe un grado de ambigüedad respecto a si se trata de una relación laboral o una "ayuda". En otro trabajo encontramos que frente a la crisis por ejemplo, el cuidado remunerado es el primer rubro recortado por parte de las familias (Herrera, 2011).

Cuando el trabajo doméstico y de cuidado no es remunerado dentro de la red, su ocultamiento es todavía mayor y aquí encontramos nuevamente una brecha impor- 
tante. En efecto, mientras el estado ecuatoriano ha insertado en sus estadísticas oficiales los usos del tiempo, visibilizando el aporte del trabajo no remunerado, en las representaciones de nuestras entrevistadas, cuidadoras en su mayoría, no se percibe una valoración del mismo, y los esfuerzos físicos y mentales que representa el quedarse a cargo del cuidado de menores o adultos mayores es verbalizado únicamente cuando se pregunta explícitamente por él. Por ejemplo, el cuidado no es parte de las discusiones sobre la decisión de migrar o no migrar, es reconocido sólo cuando significa un obstáculo para la realización de otras actividades, el estudio de las adolescentes por ejemplo, y su mercantilización no es una prioridad en la distribución de las remesas por parte de la familia que se ha quedado.

Pero no solamente que permanece oculto sino que en varias ocasiones se lo interpreta como sinónimo de la provisión de recursos materiales únicamente. Así, en varias ocasiones, sobre todo en el caso de los adultos mayores, la percepción de los familiares es que los migrantes los cuidan porque envían el dinero para sus gastos en salud o educación. Esto ha legitimado el papel de proveedoras de las mujeres migrantes. Estas son legitimadas por sus familias porque proveen de los recursos necesarios para cubrir las necesidades de la familia inmediata y en algunos casos hasta de la familia extendida, de nietas y nietos. Encontramos que en las representaciones de la gente, cuidado está muy cercano a la provisión de recursos monetarios más que a las actividades de reproducción biológica, social y cultural de las personas. Esta convergencia entre remesas y cuidados o transferencia monetarias y cuidados, oscurece aún más el trabajo no remunerado y con ello la conciencia o no de la falta de cuidados.

Por otra parte, la desconfianza frente a los servicios del estado hace que uno de los objetivos del proyecto migratorio sea garantizar educación privada para los hijos, servicios de salud privados para menores y adultos mayores y la mercantilización de las actividades de cuidado que permitan el estudio de los y las hijas que se han quedado. Es decir, las necesidades de cuidado y de reproducción social más amplia se resuelven por el lado del mercado, o, se confirma lo que ya otros estudios han señalado acerca de que la migración, y sobre todo la migración de las mujeres, vienen a llenar los vacíos y las deficiencias de los estados en garantizar la reproducción social de sus ciudadanos/a.

Finalmente, lo que el estudio muestra es que la realidad de las familias migrantes no necesariamente es específica en sus necesidades de cuidados. Por el contrario, se trata de realidades y necesidades compartidas con familias no migrantes también. En ese sentido, la migración es una válvula de escape frente a la imposibilidad de cumplir con estas expectativas y necesidades de reproducción social y una ventana desde la cual se pueden reconocer las desigualdades en la distribución de cuidado de los que se van y de los que se quedan. 


\section{Conclusiones}

Si bien la migración internacional tiene una larga tradición en el Ecuador y la región andina, la migración intercontinental de las mujeres es más reciente y tuvo un impacto mayor que las corrientes anteriores en la sociedad ecuatoriana. Este artículo muestra que las construcciones ideológicas de género están presentes en la estructuración del mercado laboral, en la forma en que se han construido las políticas sociales y en la manera en que las familias migrantes organizan su reproducción social e implementan estrategias transnacionales.

Al mirar los recursos con que cuentan las familias migrantes para el manejo de los riesgos de la reproducción social en las sociedades de origen, vemos que las remesas se constituyen no sólo en mecanismos para la subsistencia sino que también forman parte de un entramado que permite manejar los cambios en las tareas de cuidado. Así las madres o hijas migrantes se convierten en proveedoras de cuidado de sus hijos/ o padres/madres. Pero así como se produce esta mayor valoración a través de las remesas, esto contribuye a ocultar el trabajo no remunerado o mal remunerado que se ejerce al interior de estos hogares. Así, los mecanismos a disposición de las familias para el manejo de los riesgos de desprotección social son muy débiles y no han sido modificados por la migración. La presencia del estado es también muy débil en estos sectores, debido a las priorización de políticas focalizadas en los más pobres, y las prácticas de cuidado son débilmente mercantilizadas en estas familias.

Pero además, el papel de las familias transnacionales en la organización social de los cuidados es poco reconocida por las mismas familias, por las comunidades y por el estado. Por otra parte, los arreglos sociales del cuidado también están inmersos en construcciones sociales y de género que producen graves desigualdades entre los miembros de la familia. Sin embargo, la persistencia de estas prácticas y la vivencia transnacional de estas familias inclusive como un hecho inter generacional han colocado nuevas realidades tanto en el imaginario social como en el estado. Si bien todavía no hay una respuesta por parte del estado con políticas claras, es indudable que estas experiencias están paulatinamente cambiando las formas de concebir a las familias y legitimando a las mujeres migrantes en su rol de proveedoras. Resta por ver si esto significará en el mediano plazo también un cambio en la desigualdad distribución de las actividades de cuidado, no sólo entre los géneros sino también entre generaciones y clases sociales. Esto será posible únicamente si el estado empieza también por deconstruir en la base de la enunciación de sus políticas de protección social y su puesta en marcha la desvalorización de las actividades de cuidado. En ese sentido, las prácticas transnacionales de cuidado de las familias migrantes pueden constituir una semilla para transformar las ideologías fijas de género y del cuidado que aún permean las formas hegemónicas en las que se interpreta la experiencia de la migración de las mujeres en el Ecuador. 


\section{Bibliografía}

Ariza, M. (2000). Ya no soy la que dejé atrás...mujeres migrantes en República Dominicana. México: Instituto de Investigaciones Sociales - Editorial Plaza y Valdés.

Armas, A. (2008). Empleo Público en el Ecuador: una mirada desde el género. Quito: Consejo Nacional de las mujeres - Fundación Friedrich Ebert, Instituto Latinoamericano de Investigaciones Sociales - Internacional de Servicios Públicos - Secretaria Nacional Técnica de Desarrollo de Recursos Humanos y Remuneraciones del sector público. Documento de trabajo.

Bakker, I. \& Rachel S. (eds.) (2008). Beyond States and Markets: The Challenges of Social Reproduction. New York: Routledge.

Bakker, I. \& Stephen G. (2003). Power, Production and Social Reproduction. Human Insecurity in the Global Political Economy. Toronto: Palgrave -MacMillan.

Bastia, T. (2009). "Women's migration and the crisis of care: grandmothers caring for grandchildren in urban Bolivia" en Gender \& Development, Vol. 17, No. 3, pp. 389-401.

Benería, L. (2008). "The Crisis of Care, International Migration and Public Policy" en Feminist Economics, Vol. 14, No. 3, pp. 1-21.

Camacho, G. (2009). Mujeres Migrantes. Trayectoria Laboral y perspectiva de desarrollo humano. Quito: CLACSO - ABYA YALA - IEE.

Carrillo, M. C. \& Cortés A. (2008). "Por la migración se llega a Ecuador". En Herrera, Gioconda \& Jacques Ramírez (coord.), América Latina Migrante: estados, Familias, identidad. Quito: FLACSO - Ministerio de Cultura.

Cerrutti, M. (2009). "Gender and Intra-Regional Migration in South America" UNDP, Human Development Reports, Research Paper.

Egüez, P. (2005). "Mujeres y población” En Prieto, Mercedes (coord.) Mujeres ecuatorianas: entre las crisis y las oportunidades 1990-2004. Quito: FLACSO Ecuador.

Ehrenreich, B. \& Russell Hochschild A. (2003). Global Woman: Nannies, Maids and Sex Workers in The New Economy. New York: Henry Holt and Company - LLC.

Grupo Faro (2007). Cómo va la Inversión Social, Presupuesto Inicial del Estado 2007. Junio del 2007, Nro. 19. Quito: Grupo FARO-UNICEF. 
Genta, N. (2011). Abuelas ecuatorianas en las cadenas globales de cuidado ECUADOR - ESPAÑA. Maestría en Ciencias Sociales con mención en Género y Desarrollo. Quito: FLACSO.

Genta, N. \& Contreras J. (2010). "La organización social de los cuidados y vulneración de derechos en Ecuador". Santo Domingo: INSTRAW-FLACSO [En línea]URL:http://www.uninstraw.org/data/media/documents/publications/cuidado s_y_vul_de_derechos-_ecuador_.pdf.

Herrera, G. (2008). "States, Work, and Social Reproduction through the Lens of Migrant Experience: Ecuadorian Domestic Workers in Madrid" En: Bakker, Isabella \& Rachel Silvey (eds.) Beyond States and Markets: The Challenges of Social Reproduction. New York: Routledge.

Herrera, G. (2011). "La construcción de la familia migrante en las políticas públicas en Ecuador: de símbolo de la tragedia a objeto de intervención”. En FeldmanBianco, Bela, Liliana Rivera, Carolina Stefoni y Marta Villa (coord.) La construcción social del sujeto migrante en América Latina. Quito: FLACSOCLACSO- Universidad Alberto Hurtado.

Hinojosa, A. (2009). Buscando la vida: familias bolivianas transnacionales en España. La Paz: CLACSO-Fundación PIEB.

Hondagneu-Sotelo, P. (2001). Doméstica. Inmigrant Workers of Cleaning and caring in the Shadows of Affluence. Los Angeles: University of California Press.

INEC, Instituto Nacional de Estadísticas y Censos (2007). Encuesta de Empleo, Desempleo y Subempleo (ENEMDU), diciembre 2007.

INEC, Instituto Nacional de Estadísticas y Censos (2006). Encuesta de Condiciones de Vida (ECV 2006). Módulo de migración. Quinta ronda.

INEC, Consejo Nacional de las Mujeres (2007). Encuesta Nacional de Uso del tiempo (EUT) 2007.

Kusnesof, E. (1989). "A History of Domestic Service in Spanish America, 14921980". En Chaney, Elsa \& Mary Castro, Muchachas No More. Househokld Workers in Latin America and the Caribbean. Philadelphia: Temple University Press.

Lagomarsino, F. (2007). "Un ejemplo de familias transnacionales" en Lagomarsino, F. y Andrea Torre, El éxodo ecuatoriano a Europa. Quito: Abya Yala. 
Magliano, M. J. (2007). "Migración de mujeres bolivianas hacia Argentina: cambios y continuidades en las relaciones de género" En Amérique Latine Histoire et Mémoire. Les Cahiers ALHIM , No. 14, [En línea] URL : http://alhim.revues.org/index2102.html.

Martinez Franzoni, J. (2008). Domesticar la incertidumbre en América Latina Mercado laboral, politica social y familias. San José: Universidad de Costa Rica.

Martínez Buján, R. (2011). "La reorganización de los cuidados familiares en un contexto de migración internacional" en Cuadernos de Relaciones Familiares. Vol. 29, No. 1. pp. 93-123.

Marco, F. (2006). "El cuidado de la niñez en Bolivia y Ecuador: derecho de algunos, obligación de todas". Serie Mujer y Desarrollo, No 89. Santiago de Chile: CEPAL. Documento de trabajo.

Pedone, C. (2006). Estrategias migratorias y poder. Tú siempre jalas a los tuyos. Quito: Abya Yala - AECI/Plan Migración, Comunicación y Desarrollo.

Pellegrino, A. (2003). "La migración internacional en América Latina y el Caribe: tendencias y perfiles de los migrantes" Series Población y Desarrollo. No. 35. Santiago: CELADE- IDB.

Pérez Orozco, A. (2009). Cadenas globales de cuidado: ¿qué derechos para un régimen global de cuidados? Santo Domingo: INSTRAW. Documento de trabajo.

Pribilsky, J. (2007). La Chulla Vida. Gender, Migration and the Family in Andean Ecuador and New York City. Syracuse: Syracuse University Press.

Poeschel, U. (1985). La mujer Salasaca: su situación en una época de reestructuración económico-cultural. Quito: Abya Yala.

Rosero, F. (1986). El Papel del Trabajo Femenino en las Economías Campesina Comunales Quito: PUCE, informe de investigación inédito.

Salazar Parreñas, R. (2001). Servants of Globalization: Women, Migration and Domestic Work. Standford: Standford University Press.

Stefoni, C. (2002). "Mujeres Inmigrantes Peruanas en Chile" en Papeles de Población, Vol. Julio-Septiembre, No. 33, pp.118-145. México: Universidad Autónoma de México. 
Vásconez, A., Armas, A. \& Contreras, J. (2009). "Estudio del cuidado en Ecuador. Trabajo remunerado y no remunerado" Quito: CONAMU-INEC- UNIFEMAECI, versión preliminar no publicada.

Vega Solís, C. (2009). Culturas del cuidado en transición. Barcelona: Editorial UOC.

Wagner, H. (2009). "Mujeres ecuatorianas en el empleo doméstico en Madrid. Ser para otros para beneficio mutuo". En Camacho, Gloria \& Kattya Hernández (ed.) Miradas transnacionales. Visiones de la migración ecuatoriana desde España y Ecuador. Quito: CEPLAES - SENAMI.

Williams, F. (2010). "Migration and Care: themes, Concepts and Challenges" en Social Policy and Society, Vol.9, No. 3, pp. 385-396. Cambrigde: Cambrigde Universtiy Press.

Yeats, N. (2005). "Global Care Chains: a Critical Introduction" en Global Migration Perspectives, No. 44. Ginebra: Global Comission on International Migration. 